\title{
Micromachined Thermal Shear-Stress Sensor for Underwater Applications
}

\author{
Yong Xu, Member, IEEE, Qiao Lin, Member, IEEE, Guoyu Lin, Rakesh B. Katragadda, Fukang Jiang, \\ Steve Tung, Member, IEEE, and Yu-Chong Tai, Senior Member, IEEE
}

\begin{abstract}
This paper reports the development of micromachined thermal shear-stress sensors for underwater applications. The thermal shear-stress sensor is a polysilicon resistor sitting atop a vacuum-insulated nitride diaphragm. Special challenges for underwater measurements, such as the waterproof coating and minimization of pressure crosstalk, have been addressed. More rigid diaphragms than the aerial sensors are implemented to increase the operating range and reduce pressure crosstalk, with the cost of larger power consumption and lower sensitivity. Sensors with different diaphragm dimensions and resistor lengths have been fabricated and tested. Nearly zero pressure sensitivity has been achieved by either reducing the diaphragm width or adjusting the sensing element length. The effects of overheat ratio and operating mode on the sensor's pressure crosstalk have been discussed. Parylene $C$ is chosen as the waterproof material for the underwater shear-stress sensors. The primary failure mode is identified as the corrosion of the soldering pads.

[1385]
\end{abstract}

Index Terms-Microsensor, thermal flow sensor, underwater sensor.

\section{INTRODUCTION}

W ALL shear-stress measurement is of crucial importance for many fluid dynamic monitoring and diagnostics applications [1]. For example, we have developed shear-stress sensors for active drag reduction [2], [3] and for flow separation detection of unmanned aerial vehicles [4]-[6]. The wall shear stress can be determined directly using floating element method [7]-[12]. Alternatively, it can be measured indirectly using the Stanton tube, Preston tube, sublayer fence, or techniques that are based on electrochemical or thermal principles [13]. Among these approaches, the thermal method is most frequently used. This is mainly because the thermal method can be used in a wide variety of flows, it almost does not interfere with the flow, and it offers the possibility of measuring time-varying flows. With the MEMS technology, novel thermal shear-stress sensors have been successfully developed [5], [14]-[19]. Compared with conventional thermal shear-stress sensors that are typically made by

Manuscript received July 21, 2004; revised March 22, 2005. Subject Editor N. de Rooij.

Y. Xu and R. B. Katragadda are with the Electrical and Computer Engineering, Wayne State University, Detroit, MI 48202 USA (e-mail: yxu@ece. eng.wayne.edu).

Q. Lin is with the Mechanical Engineering, Carnegie Mellon University, Pittsburgh, PA 15213 USA.

G. Lin is with the ANSYS, Inc., Canonsburg, PA 15317 USA.

F. Jiang is with the Umachines, Inc., Altadena, CA 91001 USA

$\mathrm{S}$. Tung is with the Mechanical Engineering, University of Arkansas, Fayetteville, AR 72701 USA.

Y.-C. Tai is with the Electrical Engineering, California Institute of Technology, Pasadena, CA 91125 USA.

Digital Object Identifier 10.1109/JMEMS.2005.856644 depositing thin metal film (mostly platinum or nickel) resistors on flat substrates, MEMS sensors offer higher spatial resolution, faster dynamic response, and smaller power consumption.

As we noticed, almost all the MEMS shear-stress sensors developed so far target applications in air, rather than in liquid (e.g., water). Nevertheless, there are some applications that require underwater shear-stress sensors. Examples include the flow pattern measurement of radio controlled submarines, the study of safety and arming mechanisms of torpedoes, to name a few.

This paper reports the development of vacuum-cavity insulated thermal shear-stress sensors for underwater applications. The cross sectional and top views of the underwater shear-stress sensor are illustrated in Fig. 1. The sensing element is a polysilicon resistor embedded in a nitride diaphragm with a vacuum cavity underneath. During operation, the polysilicon resistor is heated electrically. The power loss of the resistor is a function of the wall shear stress $\tau$ of the ambient fluid, which is defined by

$$
\tau=\left.\mu \frac{d U}{d y}\right|_{y=0}
$$

where $\mu$ is the fluid viscosity, $U$ is the streamwise velocity and $y$ axis is normal to and originates at the sensor surface. With the vacuum cavity, heat loss to the substrate is significantly reduced and the hot surface is confined to a small area, leading to a much better spatial resolution. Although the sensor structure is very similar to aerial shear-stress sensors previously developed [14], [16], there are several challenges the underwater sensors need to address. The first challenge is the development of a compatible waterproof coating to enable the underwater operation for a certain amount of time, e.g., one month. The second challenge is the minimization of the pressure crosstalk of the sensor. For aerial applications, the pressure crosstalk is not a serious problem since the air pressure variation is negligible in most cases. However, the water pressure exerted on the sensor can change significantly, e.g., during a submarine dive. In addition, the pressure range of underwater shear-stress sensors needs to be large enough to allow deep-water operations.

\section{DESIGN}

\section{A. Waterproof Coating}

For waterproof coating, low-temperature oxide (LTO) was originally investigated, but then abandoned because eventually the sensors will be integrated onto flexible skins to enable themeasurement of shear stress on nonplanar surfaces. The flexible skin technology has been described elsewhere [4]-[6]. It has been demonstrated that LTO cracks easily and breaks metal interconnect wires underneath when the skin is subject to bending. In this 

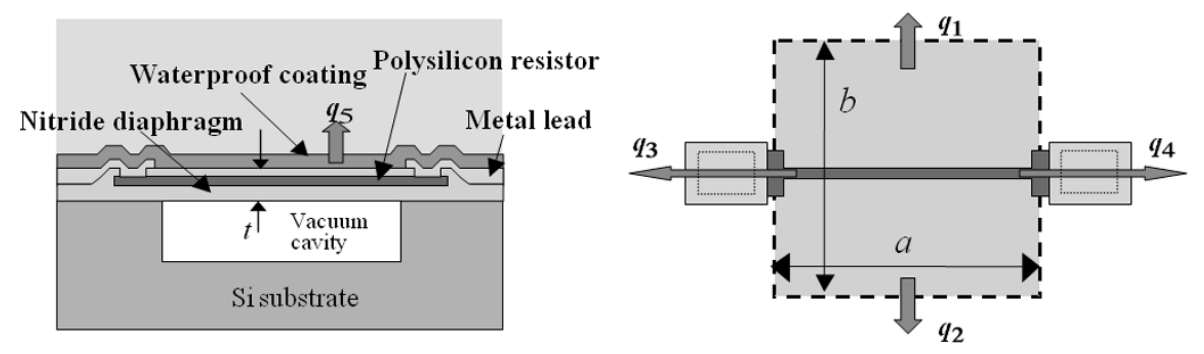

Fig. 1. Cross sectional and top views of the underwater shear-stress sensor.

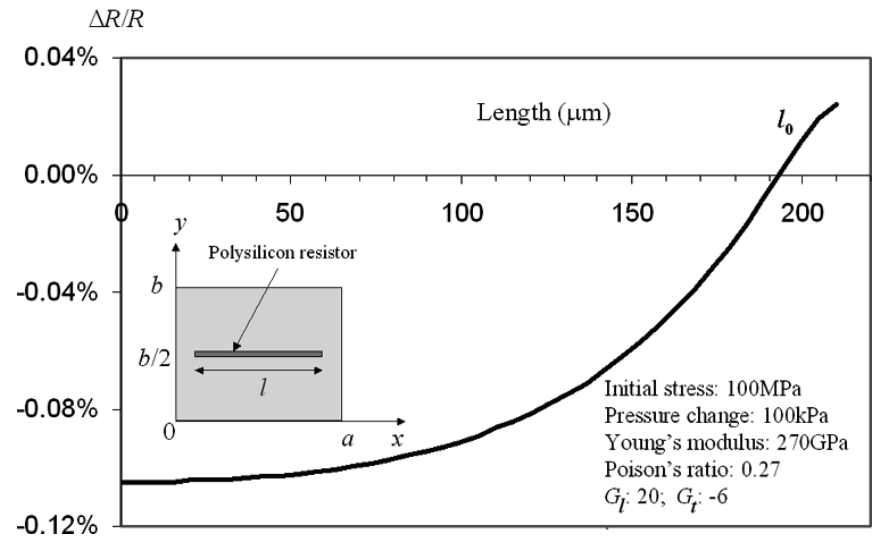

Fig. 2. Simulated relative resistance change as a function of polysilicon resistor length on a $210 \mu \mathrm{m} \times 210 \mu \mathrm{m} \times 4 \mu \mathrm{m}$ nitride diaphragm.

work, parylene $\mathrm{C}$ is chosen as the waterproof material. Parylene is the generic name for members of a unique family of thermoplastic polymers that are deposited by sublimating a dimer of para-xylylene (di-para-xylylene, or DPXN). Parylene is flexible, resistant to water transmittance, and vapor phase deposited at room temperature [20]. The three commonly used members are parylene $\mathrm{N}, \mathrm{C}$, and $\mathrm{D}$. The measured moisture vapor transmission rates (at $90 \% \mathrm{RH}, 37^{\circ} \mathrm{C}$ ) of parylene $\mathrm{N}, \mathrm{C}, \mathrm{D}$ are $1.5,0.21$, and $0.25 \mathrm{~g} \bullet \mathrm{mil} / 100 \mathrm{in}^{2} \bullet$ day, respectively, which are much smaller than those of epoxides, silicones, and urethanes [20].

\section{B. Pressure Sensitivity Analysis}

The vacuum-insulated diaphragm provides an excellent thermal isolation. On the other hand, pressure crosstalk is induced when the pressure of the ambient fluid varies. When there is a deflection of the diaphragm, the relative resistance change of the polysilicon resistor is given by

$$
\frac{\Delta R}{R}=G_{l}\left\langle\varepsilon_{l}\right\rangle+G_{t}\left\langle\varepsilon_{t}\right\rangle
$$

where $G_{l}$ and $G_{t}$ are longitudinal and transverse gauge factors of polysilicon, $\left\langle\varepsilon_{l}\right\rangle$ and $\left\langle\varepsilon_{t}\right\rangle$ are the average longitudinal and transverse strains.

If we assume the diaphragm is an ideal flat plate with clamped edge boundary and zero initial stress, a first-order analytical solution of $\left\langle\varepsilon_{l}\right\rangle$ and $\left\langle\varepsilon_{t}\right\rangle$ can be obtained [21]. Alternatively, finite element analysis has been carried out, considering the initial stress and nonideal boundary condition (i.e., the step-up anchor as shown in Fig. 4). Fig. 2 plots the simulation result of the relative resistance change on a $210 \mu \mathrm{m} \times 210 \mu \mathrm{m} \times 4 \mu \mathrm{m}$ nitride diaphragm. The width of the polysilicon is assumed to be zero.

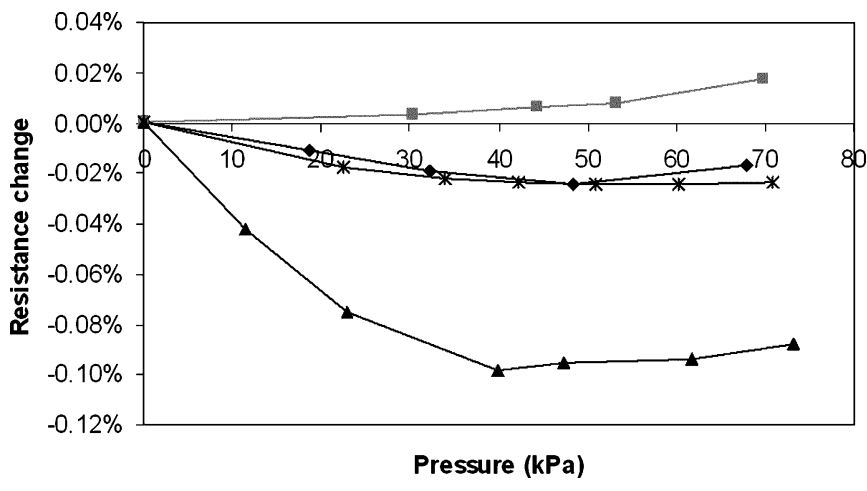

Fig. 3. Measured resistance change of four identical shear-stress sensors ( $a=$ $b=210 \mu \mathrm{m}, t=1.6 \mu \mathrm{m})$, revealing process variations.

TABLE I

DifFERENT SENSOR DESIGNS IMPLEMENTED

\begin{tabular}{c|c|c|c}
\hline Sensors & $a(\mu \mathrm{m})$ & $b(\mu \mathrm{m})$ & $l(\mu \mathrm{m})$ \\
\hline$\# 1$ & 210 & 210 & 210 \\
\hline$\# 2$ & 210 & 210 & 196 \\
\hline$\# 3$ & 210 & 210 & 180 \\
\hline$\# 4$ & 210 & 150 & 180 \\
\hline$\# 5$ & 210 & 100 & 180 \\
\hline$\# 6$ & 210 & 75 & 180 \\
\hline$\# 7$ & 210 & 45 & 180 \\
\hline
\end{tabular}

It has been found out that the resistance change of the polysilicon is not sensitive to the width as along as the width is much smaller than the diaphragm width. The longitudinal and transverse gauge factors of the heavily doped polysilicon (20 and -6) used in the simulation were reported by [22]. It can be observed that as the resistance length increases, $\Delta R / R$ approaches zero from negative values and finally becomes positive. There exists an optimal length $l_{0}$ at which the pressure sensitivity is zero. This conclusion is independent of the rigidity of the diaphragm. Thus theoretically, zero pressure crosstalk can also be achieved using the same thin diaphragm as the aerial sensor. However, the practical situation is more complicated. Fig. 3 shows the measured pressure sensitivities of four identical aerial shear-stress sensors with $210 \mu \mathrm{m} \times 210 \mu \mathrm{m} \times 1.6 \mu \mathrm{m}$ diaphragms. These four sensors, which have identical parameters such as diaphragm dimension and resistor length, were expected to have same behaviors. However, the pressure sensitivity variation can be clearly observed in Fig. 3. This sensor-to-sensor pressure sensitivity fluctuation stems from the nonideal process control and is amplified by the soft diaphragm.

Based on above observation, more rigid diaphragms are chosen for the underwater shear-stress sensors to reduce the 


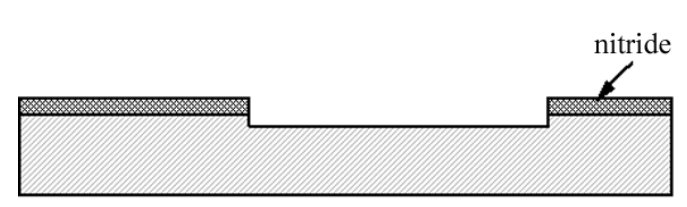

1. Deposit and pattern nitride.

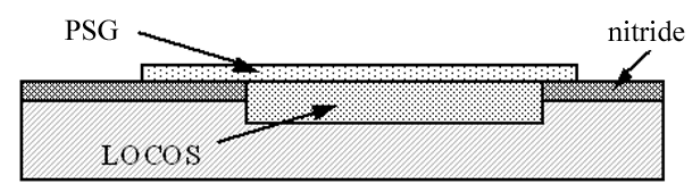

2. Local oxidation. Deposit and pattern PSG.

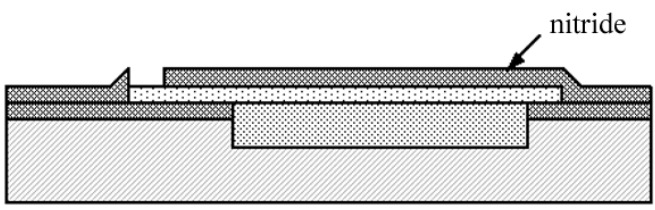

3. Deposit nitride and open etching holes.

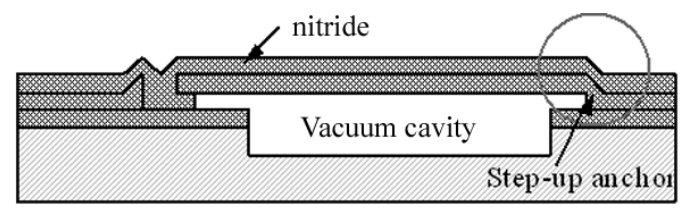

4. Remove oxide and PSG. Deposit nitride.
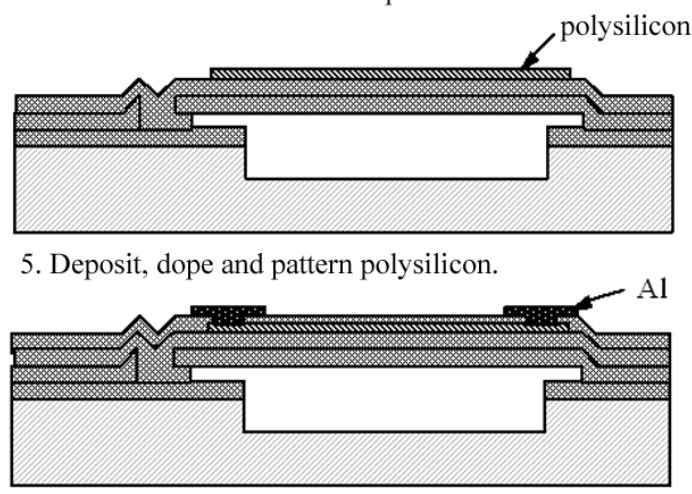

6. Deposit nitride passivation layer and A1 metalization.

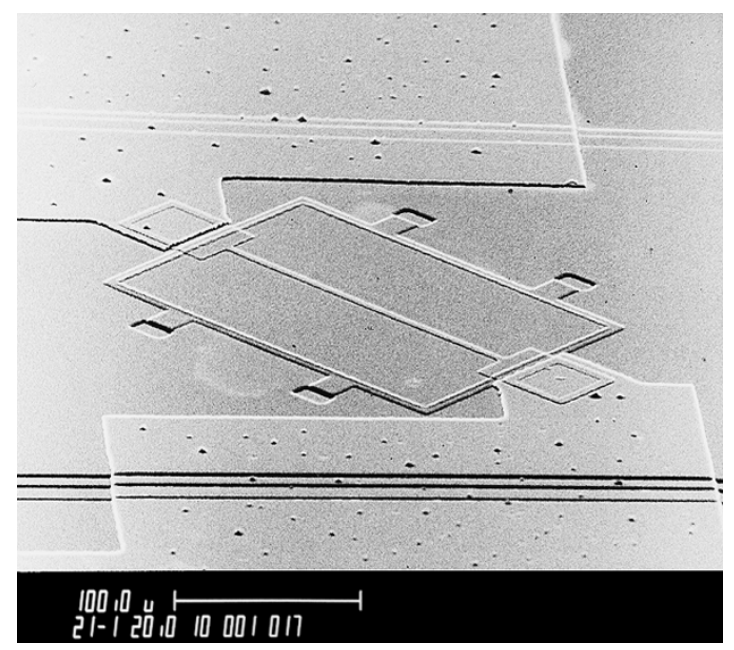

Fig. 5. SEM picture of one shear-stress sensor (design \#4 in Table I).

and the nitride diaphragms are released. Note that the step-up anchors are formed at the boundary of the diaphragm. Another nitride layer is then deposited to reach the desired diaphragm thickness and seal the cavities as well. Next, a $0.5-\mu \mathrm{m}$-thick polysilicon layer is deposited, doped (boron ion implantation with a dose of $1 \times 10^{16} / \mathrm{cm}^{2}$, resulting in a doping concentration of $\left.2 \times 10^{20} / \mathrm{cm}^{3}\right)$, annealed $\left(1050^{\circ} \mathrm{C}, 0.5 \mathrm{~h}\right)$, and patterned to form the sensing resistors. The measured TCR (temperature coefficient of resistance) of the heavily doped polysilicon is about $0.081 \% /{ }^{\circ} \mathrm{C}$. After $0.2 \mu \mathrm{m}$ nitride is deposited as the passivation layer, the contact holes are opened and a $1.5-\mu \mathrm{m}$-thick aluminum layer is sputtered, patterned, and sintered to form the interconnect lines. Fig. 5 shows the SEM picture of one shear-stress sensor and Fig. 6 illustrates two rows of the seven different sensors. Note that the sensors are placed normal to each other in order to measure the direction as well as the shear stress of the flow. 


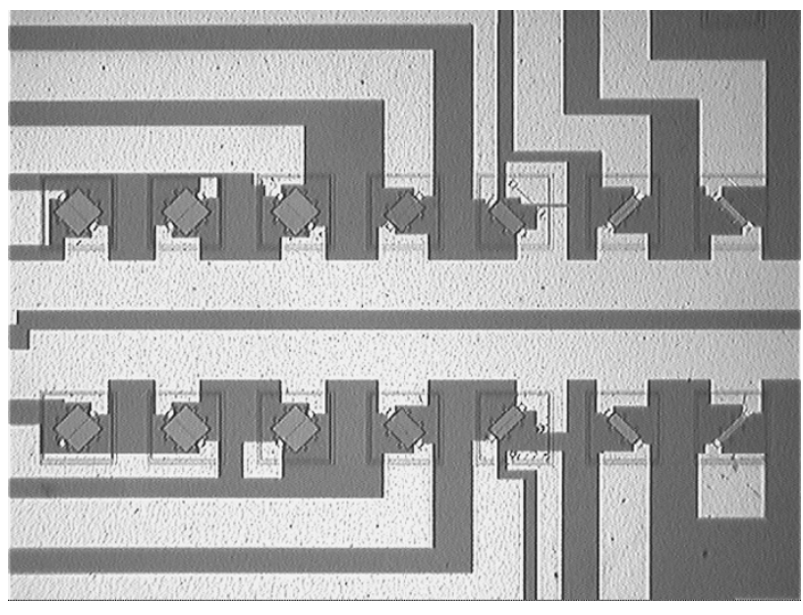

Fig. 6. Two rows of sensors with different parameters.

\section{Testing AND Discussion}

\section{A. Characterization at Zero Shear Stress}

The temperature increase versus power relationship at zero shear stress can be conveniently obtained by performing $I-V$ curve measurements, given the TCR of the sensing element. This static characterization provides valuable information of the shear-stress sensor performance.

The total heat loss of the sensor includes conduction loss to substrate $\left(q_{1}, q_{2}, q_{3}\right.$, and $\left.q_{4}\right)$ and convection loss to fluid $\left(q_{5}\right)$ as shown in Fig. 1. Radiation loss is negligible at typical operating temperature. Note that $q_{5}$ is a function of the wall shear stress of the ambient fluid and is the signal of interest. A simple way to estimate $q_{5}$ at zero shear stress is to perform the temperature-power measurements in both vacuum and fluid (air or water). Assuming in both cases the conduction loss $\left(q_{1}, q_{2}, q_{3}\right.$, and $q_{4}$ ) remains unchanged, ${ }^{1} q_{5}$ is approximately equal to the difference of the power to achieve the same temperature increase. Fig. 7 plots the temperature increase versus power curves of five sensors (sensor \#3 \#7) in vacuum and water, respectively. As expected, as the diaphragm gets smaller, more power is required to achieve the same temperature increase and less heat is transferred to water $\left(q_{5}\right)$. However, even for the sensor with $45 \mu \mathrm{m}$ wide diaphragm, there is still more than $15 \%$ of the power transferred to water.

\section{B. Shear Stress Calibration}

The shear stress calibration is conducted in a microwater tunnel. As shown in Fig. 8, the sensor chip is first bonded to a printed circuit board and then another channel chip (with a width $w$ of $2.5 \mathrm{~mm}$, a height $h$ of $0.3 \mathrm{~mm}$ and a length of $18 \mathrm{~mm}$ ) is flip-bonded on top of the sensor chip. The water flowrate $Q$ is measured by a commercial flowmeter. The distance between the first sensor and the inlet is $\sim 6 \mathrm{~mm}$, which is much larger than the entrance length of $1.29 \mathrm{~mm}$. This ensures that all the sensors being tested are in the fully developed laminar flow region.

\footnotetext{
${ }^{1}$ The conduction loss to the substrate when the sensor is in fluid is actually less than the one in vacuum. Therefore $q_{5}$ calculated by this method will be smaller than the actual value.
}

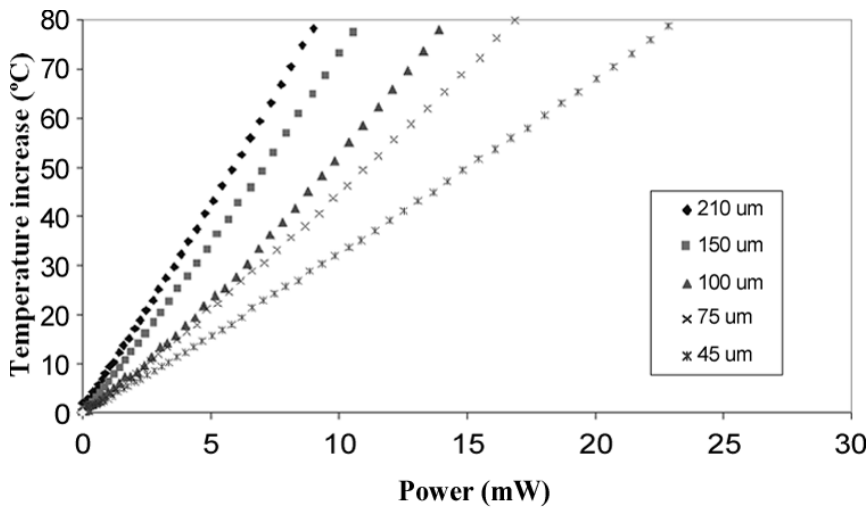

(a)

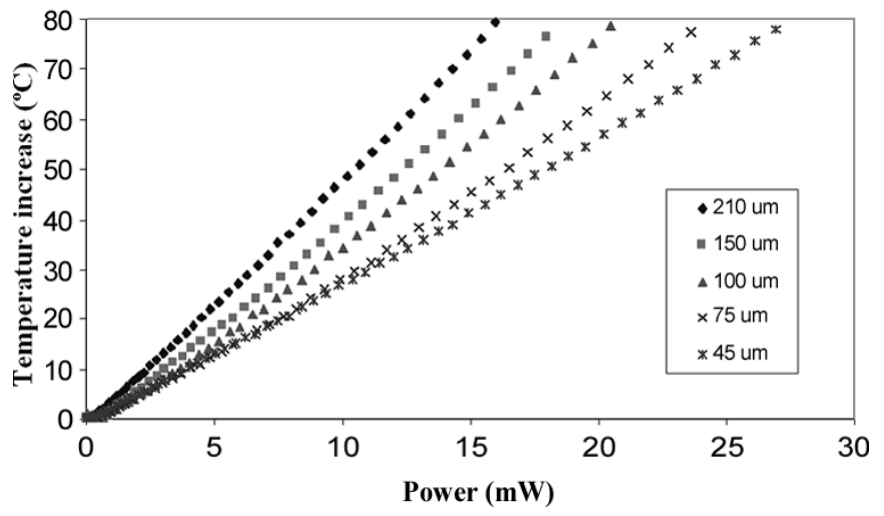

(b)

Fig. 7. Zero shear stress characteristics of five sensors with different diaphragm widths in (a) vacuum and (b) water. The temperature is calculated based on the TCR of polysilicon.

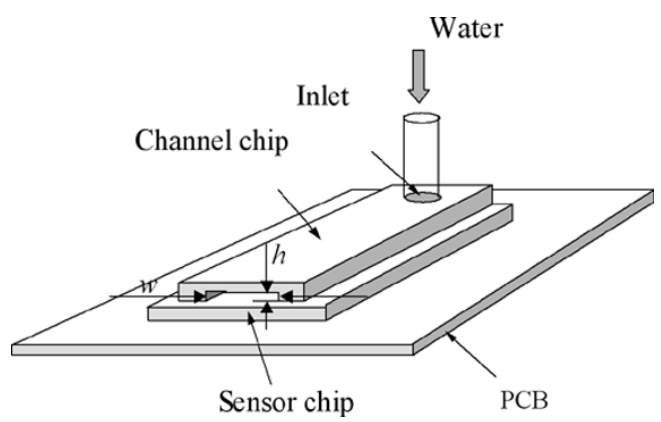

Fig. 8. A simple setup for shear stress calibration.

The shear stress can be calculated by the following equation [23]:

$$
\tau=8 \phi(n) \frac{\mu Q}{D_{h} h w}
$$

where $D_{h}$ is the hydraulic diameter, and $\phi(n)$ is a correction factor that is a function of $h / w$. For our channel, $h=0.3 \mathrm{~mm}$, $w=2.5 \mathrm{~mm}$, and $\phi(n) \approx 1.3$.

The shear-stress sensor can be biased in either constant temperature (CT) mode or constant current $(\mathrm{CC})$ mode. CT mode has higher sensitivity but the circuit is more complicated. Fig. 9 shows the simplified CT biasing circuit, where $R_{S}$ is the shear- 


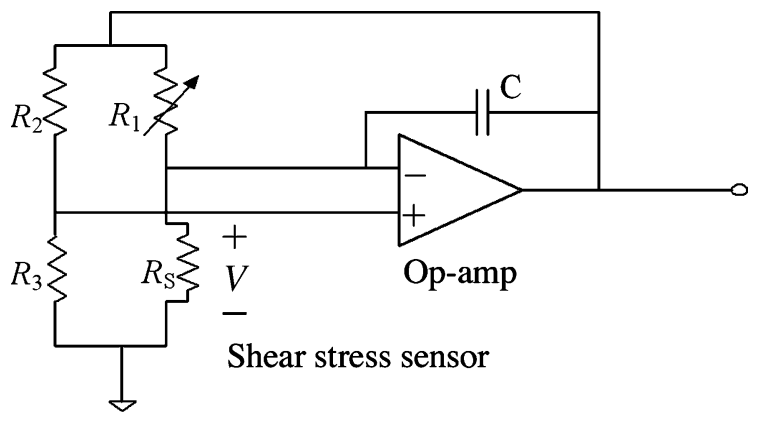

Fig. 9. Simplified constant temperature (CT) biasing circuit. $R_{\mathrm{S}}$ represents the shear-stress sensor.

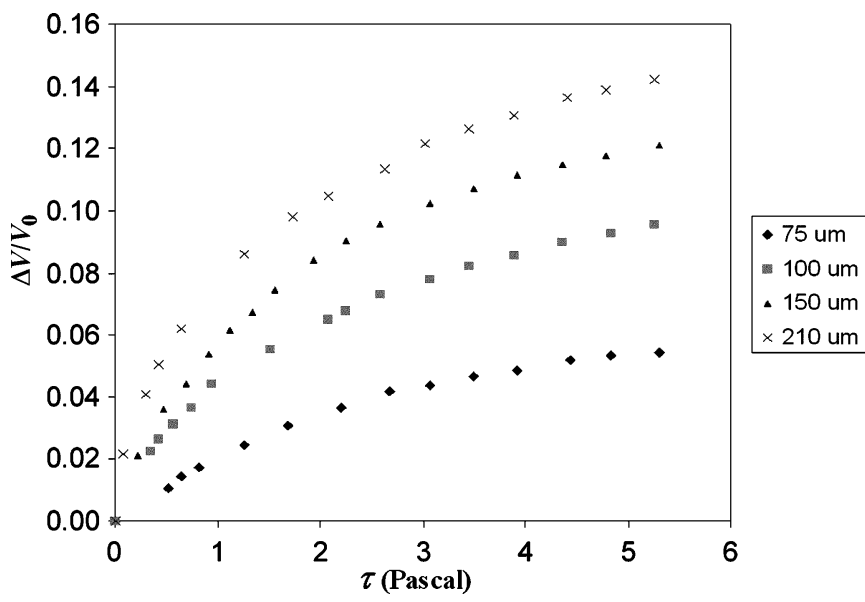

Fig. 10. Calibration curves of four sensors operate at $3 \%$ overheat ratio (sensors are $45^{\circ}$ to the flow direction).

stress sensor and $R_{1}$ is a variable resistor. An important parameter for the operation of the thermal shear-stress sensor is the (resistive) overheat ratio, which is given by

$$
a_{R}=\frac{\left(R_{S}-R_{S 0}\right)}{R_{S 0}}
$$

$R_{S 0}$ is the resistance of the shear-stress sensor at a reference temperature $T_{0}$, and $R_{S}$ is the resistance at the operating temperature $T$, which is given by

$$
R_{S}=R_{S 0}\left[1+\alpha_{T}\left(T-T_{0}\right)\right]
$$

where $\alpha_{T}$ is the TCR of the polysilicon resistor. The relationship between shear stress $\tau$ and the input power $P$ to the sensor is typically described by the following empirical equation [13]:

$$
P=\frac{V^{2}}{R_{S}}=\Delta T\left(A_{t}(\rho \tau)^{\frac{1}{n}}+B_{t}\right)
$$

where $V$ is the voltage across the shear-stress sensor, $\Delta T$ is the average temperature difference between the sensor and ambient, $\rho$ is the density of the fluid, $A_{t}, B_{t}$ and $n$ are usually determined experimentally.

Shown in Fig. 10 are the shear-stress calibration results of four sensors (\#3, \#4, \#5, and \#6) that have different diaphragm widths $(75,100,150$, and $210 \mu \mathrm{m})$. All the sensors operate at CT mode with a $3.0 \%$ overheat ratio, equivalent to a working temperature $37^{\circ} \mathrm{C}$ above ambient water. As expected, the sensor with a larger diaphragm offers higher shear-stress sensitivity. Note that the

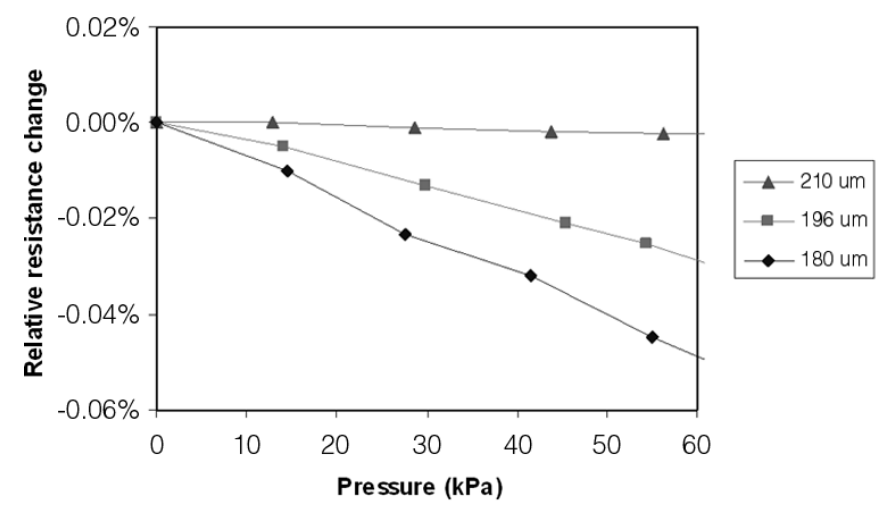

(a)

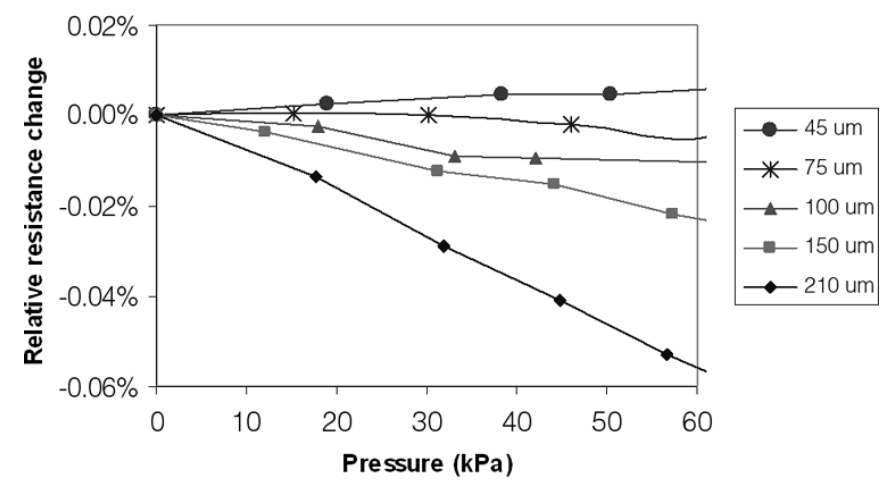

(b)

Fig. 11. Measured relative resistance changes $(\Delta R / R)$ versus pressure with (a) different sensing element lengths and (b) different diaphragm widths.

sensors are $45^{\circ} \mathrm{C}$ to the flow direction during the calibration. The maximum sensitivity, when the sensing element is normal to the flow, will be higher than what we achieved now [24].

It is worth noting that $\Delta V$ reflects the combined thermal and electrical characteristics of the sensor as illustrated by (6). In Fig. 10, the output voltage change $\Delta V$ is normalized to the static voltage $V_{0}(10-12 \mathrm{~V})$ to eliminate the effect of electrical resistance on the sensitivity [25]. After this normalization, $\Delta V / V_{0}$ only reflects the sensor's thermal characteristics. Alternatively, the power loss to water can be plotted as a function of shear stress to achieve the same goal.

\section{Pressure Sensitivity}

Fig. 11(a) shows the relative resistance change of three sensors (\#1, \#2, and \#3) with same diaphragm dimensions $(210 \mu \mathrm{m} \times$ $210 \mu \mathrm{m} \times 4 \mu \mathrm{m})$ but different resistor lengths (180, 196, and $210 \mu \mathrm{m}$, respectively). It is very interesting to note that the resistance change is almost zero when the resistor length is equal to the length of diaphragm $(210 \mu \mathrm{m})$. On the other hand, the finite element simulation predicts that the pressure sensitivity should be positive with this length (see Fig. 2). It is worth pointing out that the length $l_{0}$ at which $\Delta R / R$ is zero is not very sensitive to Young's modulus, diaphragm thickness, the initial stress, and the boundary condition of the nitride diaphragm. Instead, it is very sensitive to the gauge factors of polysilicon. Because the average longitudinal strain is zero (the linear analytical model) or almost zero (finite element method) when the resistor length is $210 \mu \mathrm{m}$, a possible interpretation is that the magnitude of the transverse 
gauge factor of the polysilicon we fabricated is smaller than the value $(-6)$ reported by [22].

Fig. 11(b) shows the measurement results of pressure sensitivity of sensors in the second group $(\# 3 \sim \# 7)$, which have same resistor length $(180 \mu \mathrm{m})$ but different diaphragm widths. It is observed that the relative resistance change of the sensor with $210-\mu \mathrm{m}$-wide diaphragm is negative and as the diaphragm width decreases, the slope approaches zero. The sensor with $75-\mu \mathrm{m}$-wide diaphragm exhibits nearly zero pressure sensitivity. The $45-\mu \mathrm{m}$-wide sensor shows positive resistance change, which is due to the dominance of the transverse strain when $b$ is much smaller than $a$.

The pressure sensitivities shown in Fig. 11 are expressed in terms of relative resistance change. But during the real operation, the pressure sensitivity exhibits in the form of voltage change. Analogous to the TCR, we can also define pressure coefficient of resistance (PCR). Therefore, we have the following expression for the resistance of the sensing element:

$$
R=R_{0}\left(1+\alpha_{T} \Delta T+\alpha_{P} \Delta P\right)
$$

where $\alpha_{P}$ is the PCR. When operating in CT mode, the negative feedback circuit actually keeps the resistance of sensing element constant. In this sense, the CT mode is more appropriately named as constant resistance (CR) mode. When there is $\Delta R$ caused by a pressure variation $\Delta P$, the temperature must change in opposite direction to compensate this change. From (7), this temperature change is given by

$$
\Delta T^{\prime}=-\frac{\alpha_{P}}{\alpha_{T}} \Delta P
$$

We use $\Delta T^{\prime}$ to denote this temperature change to distinguish it from the temperature difference between the sensor and ambient fluid. The input power of the sensor can be expressed as

$$
\frac{V^{2}}{R}=\left(A_{t}(\rho \tau)^{\frac{1}{n}}+B_{t}\right) \Delta T=C \Delta T
$$

where $C$ is a weak function of temperature and can be considered as a constant to a first order of approximation. When there is a temperature change induced by pressure variation, we have

$$
\frac{\left(V+\Delta V^{\prime}\right)^{2}}{R}=C\left(\Delta T+\Delta T^{\prime}\right)
$$

where $\Delta V^{\prime}$ is the voltage change in response to the pressure variation. Neglecting $\Delta V^{\prime 2}$ term, we have

$$
\frac{2 V \Delta V^{\prime}}{R}=C \Delta T^{\prime}=-C \frac{\alpha_{P}}{\alpha_{T}} \Delta P .
$$

Note that $C=V^{2} / R \Delta T$ based on (9). Therefore $\Delta V^{\prime}$ is given by

$$
\Delta V^{\prime}=-\frac{V}{2 \alpha_{T} \Delta T} \alpha_{P} \Delta P .
$$

The above expression can be further simplified since $\alpha_{T} \Delta T$ is the resistance overheat ratio $a_{R}$ and $\alpha_{P} \Delta P$ is the relative resistance change $(\Delta R / R)_{P}$ caused by pressure variation

$$
\Delta V^{\prime}=-\frac{V}{2 a_{R}}\left(\frac{\Delta R}{R}\right)_{P}
$$

Note that $V$ is proportional to $\sqrt{a_{R}}$. Therefore, $\Delta V^{\prime}$ is inversely proportional to $\sqrt{a_{R}}$. A larger overheat ratio $a_{R}$ is preferred to obtain lower pressure sensitivity and higher shear-stress sensitivity. However, the operating temperature cannot be too high in order to avoid the bubble generation in the water. This is another reason that the pressure crosstalk for aerial applications is not as serious as underwater ones because the overheat ratio in air can be much higher, e.g., $20 \%$.

We measured one sensor (\#3, $210 \mu \mathrm{m}$ wide diaphragm, 180 $\mu \mathrm{m}$ long sensing element) with a resistance change rate of $0.00074 \% / \mathrm{kPa}$. When operated at an overheat ratio of $3 \%$ in CT mode with an output voltage (across the sensing element) of $5.2 \mathrm{~V}$, the sensor should exhibit a pressure sensitivity of 0.64 $\mathrm{mV} / \mathrm{kPa}$ according to (13). The actual value measured is 0.74 $\mathrm{mV} / \mathrm{kPa}$, which is very close to the theoretical one. Note that the pressure sensitivity is measured at zero shear stress.

In the case of $\mathrm{CC}$ mode, the voltage change due to resistance variation is simply

$$
\Delta V^{\prime}=\left(\frac{\Delta R}{R}\right)_{p} V
$$

where $V$ is approximately proportional to $\sqrt{a_{R}}$. Therefore at CC mode, higher overheat ratio results in a larger pressure crosstalk. It is worth noting that at $\mathrm{CC}$ mode, the operating temperature of sensor is not a constant at different shear stresses. Here the overheat ratio of $\mathrm{CC}$ mode is defined at zero shear stress.

The ratio of the pressure crosstalks at $\mathrm{CT}$ mode and $\mathrm{CC}$ mode is

$$
\frac{\Delta V_{C T}^{\prime}}{\Delta V_{C C}^{\prime}}=\frac{1}{2 a_{R}} .
$$

The above equation is obtained based on the assumption that the sensors operated at CC and CT modes have the same output voltage $V$ at the specific operating points under consideration. For underwater measurement, the overheat ratio is usually small (e.g., 3\%) to avoid boiling. Therefore the pressure sensitivity of CT mode is larger than that of CC mode, although CT mode offers higher shear-stress sensitivity. Based on above first-order analysis, the authors conclude that the selection of operation mode (CT or CC) should depend on the overheat ratio $a_{R}$. If a relatively large $a_{R}$ is allowed, CT mode is usually preferred. On the other hand, if a specific application limits the $a_{R}$ to a very small value, then $\mathrm{CC}$ mode is probably a better choice.

There are many other possible ways to reduce the pressure crosstalks besides using the on-chip pressure sensor to perform pressure compensation. For example, as mentioned previously, the operating temperature of the sensor can be increased reasonably in CT mode. In terms of fabrication, the sensing element (polysilicon resistor) can be buried in the middle plane of the nitride diaphragm in the cost of making the contact-hole opening process more complicated. Examining (2), it is clear that the gauge factor of polysilicon contributes considerably to the pressure sensitivity. Therefore, it is of interest to use metal (e.g., platinum), whose gauge factor is much smaller, as the heating element. The cost is a smaller output voltage due to the lower electrical resistivity of metal.

\section{Waterproof Testing}

Preliminary underwater tests show that, when operated at $55^{\circ} \mathrm{C}$, sensors coated with $2 \mu \mathrm{m}$ parylene $\mathrm{N}$ can survive in 


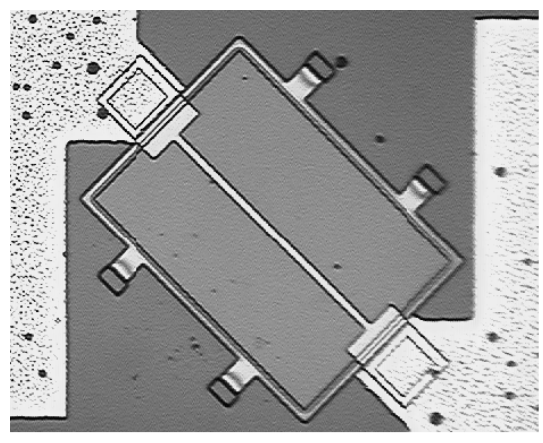

(a)

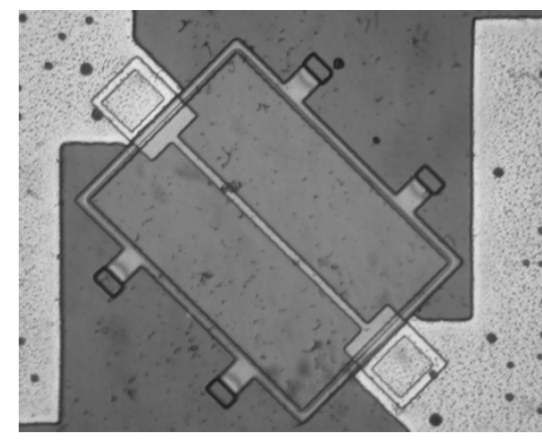

(b)

Fig. 12. Micrographs of a parylene C-coated shear-stress sensor (a) before and (b) after 27 months saline soaking.

water at least for one month. Tests of sensors coated with parylene $\mathrm{C}$, which has even smaller moisture vapor transmission rate [20], have also been performed. After soaking in saline solution for 27 months at room temperature, the sensors coated with $2 \mu \mathrm{m}$ parylene $\mathrm{C}$ still function properly. As shown in Fig. 12, no severe degradation of parylene $\mathrm{C}$ is observed. The integrity of the parylene $\mathrm{C}$ waterproof coating has also been confirmed using electrical measurement. It is worth pointing out that although sensors and aluminum wires coated with parylene $\mathrm{C}$ are well-protected, severe corrosion is observed on soldering pads where the lead wires connect to the PCB. This is consistent with our previous observation and we conclude that the primary failure mechanism is the breakdown of the soldering pads. However, this is not a big concern when eventually the rigid-chip sensors is made flexile using a unique flexible skin technology described in [4], [5]. With the flexible skin structure, the bonding pads of the skin can be bent to the back side of the package through a slit and all the bonding/soldering will be performed on the back side where is completely isolated from the water. Therefore, the flexible underwater shear-stress sensor skins will not only enable the measurement of shear stress on nonplanar surfaces, but also improve the reliability significantly. It is also worth noting that the pressure variation underwater may cause delamination of the parylene layer. To address this issue, the sensor is treated with a dilute isopropyl alcohol-water solution of the organic silane gamma-methacryloxypropyltrimethoxysilane (A-174) before parylene deposition to significantly improve the adhesion [20].

\section{SUMMARY}

Micromachined thermal shear-stress sensors for underwater applications have been successfully developed. Compared with aerial shear-stress sensors, the underwater sensors employ more rigid diaphragms, which lead to larger power loss to the substrate. Shear-stress sensitivity of sensors with different diaphragm dimensions has been characterized in a fully developed laminar flow inside a microchannel. It has been demonstrated that the sensor with larger diaphragm exhibits higher sensitivity. Adjusting either the diaphragm width or the polysilicon resistor length can minimize the pressure sensitivity. A more rigid diaphragm is preferred to achieve a better controllability, higher yield and wider operation range. The impact of overheat ratio on the sensor's pressure sensitivity has been analyzed.
For CT mode, large overheat ratio is preferred to achieve low pressure sensitivity and high shear-stress sensitivity. For CC mode, both shear-stress sensitivity and pressure sensitivity will be higher as the overheat ratio increases. There are several ways to further reduce the pressure sensitivity. For example, the operating temperature can be increased, the sensing element can be buried in the middle plane of the nitride diaphragm, or platinum can be used due to its small gauge factor. Parylene has proven to be a satisfactory waterproof material for our applications. Soldering pads are identified as the weakest point to fail. This failure mode can be eliminated by making flexible underwater shear-stress sensor skins, which also enable the measurement on nonplanar surfaces.

\section{REFERENCES}

[1] C. M. Ho and Y. C. Tai, "Review: MEMS and its applications for flow control," Trans. ASME-J. Fluids Eng., vol. 118, pp. 437-447, 1996.

[2] T. Tsao, F. Jiang, C. Liu, R. Miller, S. Tung, J.-B. Huang, B. Gupta, D. Babcock, C. Lee, Y.-C. Tai, C.-M. Ho, J. Kim, and R. Goodman, "MEMS-based active drag reduction in turbulent boundary layers," in Microengineering Aerospace Systems, H. Helvajian, Ed: The Aerospace Press, 1999, pp. 553-580.

[3] X. Q. Wang, Z. Han, F. Jiang, T. Tsao, Q. Lin, Y. C. Tai, and C. M. Ho, "A fully integrated shear stress sensor," in Proc. International Conference on Solid-State Sensors and Actuators (Transducer), Sendai, Japan, Jun. 7-10, 1999, pp. 1074-1077.

[4] Y. Xu, Y.-C. Tai, A. Huang, and C.-M. Ho, "IC-integrated flexible shearstress sensor skin," J. Microelectromech. Syst., vol. 12, pp. 740-747, 2003.

[5] Y. Xu, F. Jiang, S. Newbern, A. Huang, C.-M. Ho, and Y.-C. Tai, "Flexible shear-stress sensor skin and its application to unmanned aerial vehicles," Sens. Actuators A: Phys., vol. 105, pp. 321-329, 2003.

[6] F. Jiang, Y. Xu, T. Weng, Z. Han, Y.-C. Tai, A. Huang, C.-M. Ho, and S. Newbern, "Flexible shear stress sensor skin for aerodynamics applications," in Proc. IEEE International Conference on Micro Electro Mechanical Systems (MEMS), Miyazaki, Japan, Jan. 23-27, 2000, pp. 364-369.

[7] A. Padmanabhan, H. D. Goldberg, K. S. Breuer, and M. A. Schmidt, "Silicon micromachined floating-element shear-stress sensor with optical position sensing by photodiodes," in Proc. International Conference on Solid-State Sensors and Actuators, and Eurosensors IX, Stockholm, Sweden, Jun. 25-29, 1995, pp. 436-439.

[8] T. Pan, D. Hyman, M. Mehregany, E. Reshotko, and B. Willis, "Characterization of microfabricated shear stress sensors," in Proc. International Conference on Solid-State Sensors and Actuators and Eurosensors IX, Stockholm, Sweden, 1995, pp. 443-446.

[9] M. A. Schmidt, R. T. Howe, S. D. Senturia, and J. H. Haritonidis, "Design and calibration of a microfabricated floating-element shear-stress sensor," IEEE Trans. Electron Devices, vol. 35, pp. 750-757, 1988.

[10] J. Shajii, K.-Y. Ng, and M. A. Schmidt, "A microfabricated floating-element shear stress sensor using wafer-bonding technology," J. Microelectromech. Syst., vol. 1, pp. 89-94, 1992. 
[11] A. V. Desai and M. A. Haque, "Design and fabrication of a direction sensitive MEMS shear stress sensor with high spatial and temporal resolution," J. Micromech. Microeng., vol. 14, pp. 1718-1725, 2004.

[12] J. Zhe, V. Modi, and K. R. Farmer, "A microfabricated wall shear-stress sensor with capacitative sensing," J. Microelectromech. Syst., vol. 14, pp. $167-175,2005$.

[13] T. J. Hanratty and J. A. Campbell, "Measurement of wall shear stress," in Fluid Mechanics Measurements, 2nd ed, R. J. Goldstein, Ed: Taylor and Francis, 1996, pp. 575-648.

[14] C. Liu, Y. C. Tai, J. B. Huang, and C. M. Ho, "Surface micromachined thermal shear stress sensor," in Proc. ASME International Mechanical Engineering Congress and Exposition, Chicago, IL, Nov. 6-11, 1994, pp. $9-15$.

[15] J. B. Huang, S. Tung, C. M. Ho, C. Liu, and Y. C. Tai, "Improved micro thermal shear-stress sensor," IEEE Trans. Instrum. Measure., vol. 45, pp. 570-574, 1996.

[16] F. Jiang, Y. C. Tai, B. Gupta, R. Goodman, S. Tung, J. B. Huang, and C. M. Ho, "A surface-micromachined shear stress imager," in Proc. IEEE International Conference on Micro Electro Mechanical Systems (MEMS), San Diego, CA, Feb. 11-15, 1996, pp. 110-115.

[17] C. Liu, J. B. Huang, Z. J. Zhu, F. K. Jiang, S. Tung, Y. C. Tai, and C. M. Ho, "Micromachined flow shear-stress sensor based on thermal transfer principles," J. Microelectromech. Syst., vol. 8, pp. 90-99, 1999.

[18] A. Cain, V. Chandrasekaran, T. Nishida, and M. Sheplak, "Development of a wafer-bonded, silicon-nitride membrane thermal shear-stress sensor with platinum sensing element," in Proc. Solid-State Sensor and Actuator Workshop, Hilton Head Island, South Carolina, June 4-8, 2000, pp. 300-303.

[19] M. Sheplak, V. Chandrasekaran, A. Cain, T. Nishida, and L. N. Cattafesta, "Characterization of a silicon-micromachined thermal shear-stress sensor," AIAA Journal, vol. 40, pp. 1099-1104, 2002.

[20] "Parylene Data Sheet, Specialty Coating System,", Indianapolis, IN.

[21] C.-T. Wang, Applied Elasticity. New York: McGraw-Hill, 1953.

[22] E. Obermeier and P. Kopystynski, "Polysilicon as a material for microsensor applications," Sens. Actuators, Phys., vol. 30, pp. 149-155, 1992.

[23] J. A. Schetz and A. E. Fuhs, Handbook of Fluid Dynamics and Fluid Machinery. New York: Wiley, 1996, vol. I.

[24] J. B. Huang, F. K. Jiang, Y. C. Tai, and C. M. Ho, "A micro-electro-mechanical-system-based thermal shear-stress sensor with self-frequency compensation," Measure. Sci. Technol., vol. 10, pp. 687-696, 1999.

[25] Y. Xu, C.-W. Chiu, F. Jiang, Q. Lin, and Y.-C. Tai, "A MEMS multisensor chip for gas flow sensing," Sens. Actuators A, Phys., vol. 121, pp. 253-261, 2005.

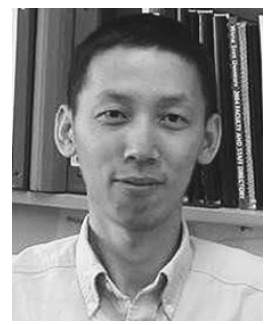

Yong Xu (M'03) received the B.S. degree in electronics engineering from Tsinghua University, Beijing, China, in 1997 and the M.S. and Ph.D. degrees in electrical engineering from the California Institute of Technology, Pasadena, in 1998 and 2002, respectively.

$\mathrm{He}$ is currently an Assistant Professor in the Department of Electrical and Computer Engineering at Wayne State University. His research interests include MEMS smart skins, intelligent textiles, microfluidics, biosensors, medical sensors, novel packaging technology, and nanotechnology.

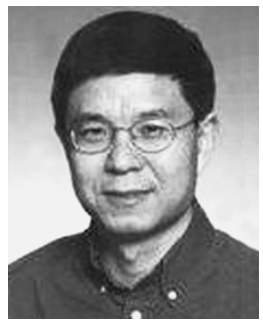

Qiao Lin (M'04) received the Ph.D. degree in mechanical engineering from the California Institute of Technology (Caltech), Pasadena, in 1998 with dissertation research on kinematics and mechanics of robotic manipulation.

He has conducted postdoctoral research in microelectromechanical systems (MEMS) at the Caltech Micromachining Laboratory from 1998 to 2000, and has since been an Assistant Professor in the Department of Mechanical Engineering at Carnegie Mellon University, Pittsburgh, PA. His research interests are in MEMS, including analysis, design and fabrication of micro/nano-fluidic, thermal, and robotic devices for biological and chemical applications.

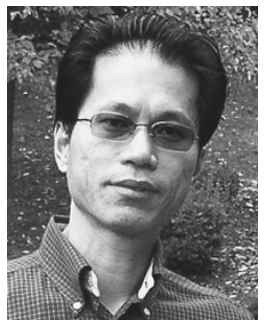

Guoyu Lin received the Ph.D. degree from Technical University Hamburg-Harburg, Hamburg, Germany, in 1997.

He is currently a Technical Fellow at ANSYS, Inc., Canonsburg, PA. He has been working in the areas of material constitutive modeling, nonlinear fracture mechanics and finite element method for over 20 years. He is also an active reviewer for the Journal of Engineering Fracture Mechanics.

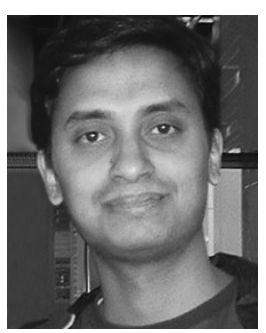

Rakesh B. Katragadda received the B.E. degree from University Visvesvaraiah College of Engineering (UVCE), Bangalore, India, in 2002 and is currently pursuing the Master's degree at Wayne state University, MI. His research interests include MEMS-based smart skin for wearable computing and biosensors for DNA hybridization.

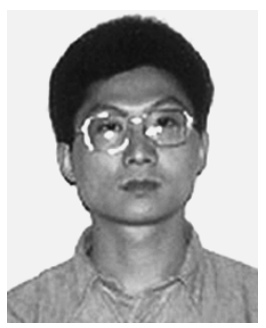

Fukang Jiang received the M.S. and Ph.D. degrees in electrical engineering from the California Institute of Technology (Caltech), Pasadena, in 1992 and 1997, respectively, where his research was on the development of MEMS flow sensors and their advanced packaging technologies such as the flexible-skin-type substrate for MEMS devices.

He has been with Umachines, Inc., developing various optical MEMS products such as optical switches and 3D scanning mirror arrays since 1997.

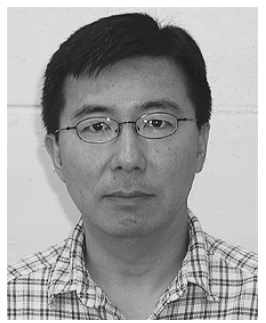

Steve Tung (M'03) received the B.S. degree from the National Taiwan University in 1984 and the Ph.D. degree in mechanical engineering from the University of Houston, TX, in 1992.

From 1993 to 1997, he worked as a Postdoctoral Fellow at the Department of Mechanical and Aerospace Engineering at the University of California (UCLA), Los Angeles. From 1997 to 1999, he was an Engineering Specialist at Litton Guidance and Control Systems at Woodland Hills, CA. In January 2000, he joined the Department of Mechanical Engineering at the University of Arkansas where he is currently an associate professor. His research interests include microfluidics, hybrid flagellar motor systems, and carbon nanotube based devices. He has served as the principal investigator of several research projects funded by NSF, NASA, and ONR. In 2001 and 2003, he was named the University of Arkansas College of Engineering Outstanding Researcher in Mechanical Engineering.

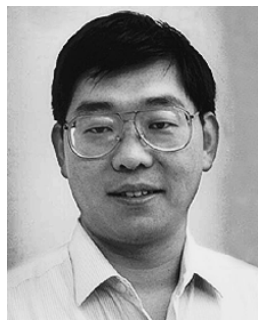

Yu-Chong Tai (M'97-SM'03) received the B.S. degree from National Taiwan University and the M.S. and Ph.D. degrees in electrical engineering from the University of California at Berkeley in 1986 and 1989, respectively.

After Berkeley, he joined the faculty of Electrical Engineering at the California Institute of Technology and built the Caltech Micromachining laboratory. He is currently a full Professor of Electrical Engineering at Caltech. His research interests include flexible MEMS, integrated microfluidics, neuroprobes and chips, optical MEMS and biochemical sensors.

Dr. Tsai has received several awards such as the IBM fellowship, the Best Thesis Award, the Presidential Young Investigator (PYI) Award and the David and Lucile Packard Fellowship. He co-chaired the 2002 IEEE MEMS Conference in Las Vegas. He is currently a Subject Editor of the Journal of MEMS. He's also a Section Editor of Sensors and Actuators. 